\title{
Pelvic exenteration in gynecologic cancer: complications and oncological outcome
}

\author{
Víctor Lago ${ }^{1 *}$ (D) Ines Poveda ${ }^{1}$, Pablo Padilla-Iserte', Eduardo Simón-Sanz², Álvaro García-Granero³, \\ Jose Luis Pontones ${ }^{4}$, Luis Matute ${ }^{1}$ and Santiago Domingo ${ }^{1}$
}

\begin{abstract}
Introduction: Pelvic exenteration (PE) is indicated in cases of unresponsive, recurrent pelvic cancer or for palliative intent. Despite the fact that the surgery is associated with a high rate of morbidity, it is currently the only real option that can effect a cure.

Material and methods: Patients who underwent PE between January 2011 and July 2017 in our centre were retrospectively reviewed. Data related to surgery, complications and outcomes were recorded.

Results: Twenty-three patients were included. PE was performed due to recurrent gynaecological cancer, persistence of disease and after first diagnosis in 19 (82\%), 2 (9\%) and 2 patients (9\%), respectively. Total PE was performed in 15 cases (65\%), followed by anterior PE in 5 cases (22\%) and posterior PE in 3 cases (13\%). Early grade II, III and IV complications occurred in $15(65 \%), 5$ (22\%) and 2 patients (9\%), respectively. No mortality was observed within 30 days. Medium-late grade II, III, IV and V complications occurred in 15 (65\%), 11 (48\%), 3 (13\%) and 2 cases (9\%), respectively. Two patients died after $>30$-day period from surgery-related complications. The overall survival (OS) and disease-free survival (DFS) at 48 months after PE was $41.6 \%$ and $30.8 \%$ respectively.
\end{abstract}

Conclusions: PE provides about a $40 \%$ 4-year survival chance in a selected group of patients. The earlycomplications rate and 30-day mortality were acceptable. Nevertheless, the medium-late complication grades II-V were $65,48,18$ and 9\%, respectively. We must focus on identifying those patients who could potentially benefit most from PE.

Keywords: Pelvic exenteration, Relapsed cancer, Complications, Survival, VRAM

\section{Introduction}

Pelvic exenteration (PE) is indicated in cases of unresponsive disease, recurrent pelvic cancer or for treatment with palliative intent. Despite the high rate of associated morbidity, it is currently the only real option for cure $[1,2]$. In gynaecology, this type of surgery is performed in patients who suffer from tumours of the cervix, vulva or vagina, being less commonly performed in patients with ovarian or endometrial cancer.

Technical and scientific advances have improved PE outcomes. Some of the most significant of these advances are the options for urinary diversion and the use of flaps for vaginal reconstruction [3]. General improvements,

\footnotetext{
* Correspondence: victor.lago.leal@hotmail.com

'Department of Gynecologic Oncology, University Hospital La Fe, Avinguda de Fernando Abril Martorell, 106, 46026 Valéncia. F Tower; 3rd Floor, Valencia, Spain

Full list of author information is available at the end of the article
}

such as antibiotic treatments, thromboembolic prophylaxis and new vessel-sealing devices, have also contributed to the improvement in PE results.

Despite all these developments, however, PE's high levels of associated morbidity remain a concern, since the 5 -year survival rate has been reported as $30-60 \%$ [1]. Moreover, it is a life-challenging surgery, leading to major changes that affect patient self-image. Physical, sexual and psychosocial functions are also negatively affected by the procedure [4].

The identification of markers correlated with good results from this surgery may result in a better focus and improve the selection of patients who would benefit most from PE. Some authors have proposed that the presence of positive surgical margins, lymph node involvement or perineurial invasion be regarded as bad prognosis factors [1]. However, such factors have a 
limited utility as they can only be identified after performance of PE.

The aim of this paper is to analyse the surgical results, complications and oncological outcomes of PE. To this end, we present the results of patients who underwent this surgery in our centre (University Hospital La Fe, Spain) between January 2011 and July 2017.

\section{Material and methods}

After obtaining the approval of the local ethics committee (local code 2017/0259), all medical records of patients who underwent PE for gynaecological cancer at the Department of Obstetrics and Gynaecology at University Hospital La Fe, Spain, between January 2011 and July 2017 were assessed for inclusion. All patients underwent preoperative imaging for staging (either full body-CT or PET-CT) and were discussed by a multidisciplinary tumour board. Biopsy confirmation of disease was obtained preoperatively. PE was considered for palliative purposes only where symptoms limited the quality of life to an extreme extent.

The surgical approach was multidisciplinary; the procedure was led by a gynaecological oncologist working together with a urologist, colorectal surgeon, plastic surgeon or traumatologist as required.

Twenty-six patients were identified. Three were excluded because a partial cystectomy with ureteral re-implant was performed instead of a total cystectomy. For the remaining 23 patients who were included, PE was classified depending on which structures were resected: anterior PE (APE) if resection of the female genitals was performed as a single specimen with the lower urinary tract (bladder and urethra), posterior $\mathrm{PE}$ (PPE) if the resection of the female genitals was performed together with rectosigmoid and total PE (TPE) in cases of resection of lower urinary tract, female genitals and rectosigmoid. The exenteration groups were also sub-classified into type I (supralevator), type II (infralevator) and type III (with vulvectomy) [5].

The following details were recorded: presentation of disease, age, body mass index (BMI), Eastern Cooperative Oncology Group (ECOG) performance status, comorbidity, previous radiotherapy (RT) or chemotherapy (ChT) treatment, previous oncological surgery, origin of tumour, operative risk score of the American Society of Anesthesiologists (ASA) and the operative intention.

The following surgical variables were described: type and subtype of PE, operative time, type of stoma, urinary diversion, lymph node dissection, additional surgical procedures, intraoperative complications, units of red blood transfused, estimated blood loss, reconstructive surgery and margins (classified as not macroscopically detectable (R0) or macro-/microscopically detectable (R1)). Also recorded were all early complications (those which occurred as inpatient and/or $<30$ days after surgery) and medium-late complications (those which occurred after discharge and/or $>30$ days after surgery), length of hospital stay, time spent in the ICU and any need for re-surgery.

Complications were graded according to the Clavien-Dindo Classification [6]. Grade I complications were not reported in this study. For each patient, only the highest complication grade was recorded where a complication clearly occurred as a consequence of a prior complication of a lower grade. Complications of the same grade that were not directly related to each other were recorded separately. A specific complication that re-occurred was only recorded once.

Postoperative mortality within 30 days was defined as death regardless of cause. OS was defined as time from $\mathrm{PE}$ to death, regardless of cause and recurrence status. The second survival parameter, DFS, was defined as time from $\mathrm{PE}$ to recurrence, progression or death. For both parameters, the median time survival and the 6-month, 1-year, 2-year and 4-year survival rate were reported.

All patients were followed from the day after the PE until July 2017, or until death. They were visited at least every 3 months in the first 2 years and then every 6 months until the fifth year and annually after this date.

Kaplan-Meier survival curves were calculated (CI 95\%), for overall and disease-free survival. Statistical analysis was performed using the SPSS program (SPSS, Chicago, IL, USA).

\section{Results}

\section{Characteristics of patients and tumours}

Complete information was available for all 23 patients. As regards the presentation of disease, PE was performed in 19 cases (82\%) due to recurrent gynaecological cancer, in 2 cases (9\%) the patients were not free of disease after primary chemo-radiotherapy and PE was performed due to persistence of disease, and in the other 2 patients (9\%) the cancer was diagnosed for the first time: 1 patient presented a $10 \mathrm{~cm}$ tumour vulvar cancer involving the anus (she refused chemo-radiotherapy as a first treatment option) and the other one presented a cervical cancer diagnosed after delivery in association with a large metastasis located in the episiotomy scar site (Fig. 1).

Demographic, preoperative and tumour data are summarized in Table 1. The mean age at diagnosis was 57.8 years (range 29-84) with most of the patients having pre-menopausal status $(74 \%)$. The vast majority of the patients received chemo-radiotherapy $(70 \%)$ or RT (17\%). Previous oncological surgery had been performed in almost $78 \%$ of the patients. The primary tumour origin was the cervix in 11 patients (48\%), followed by 


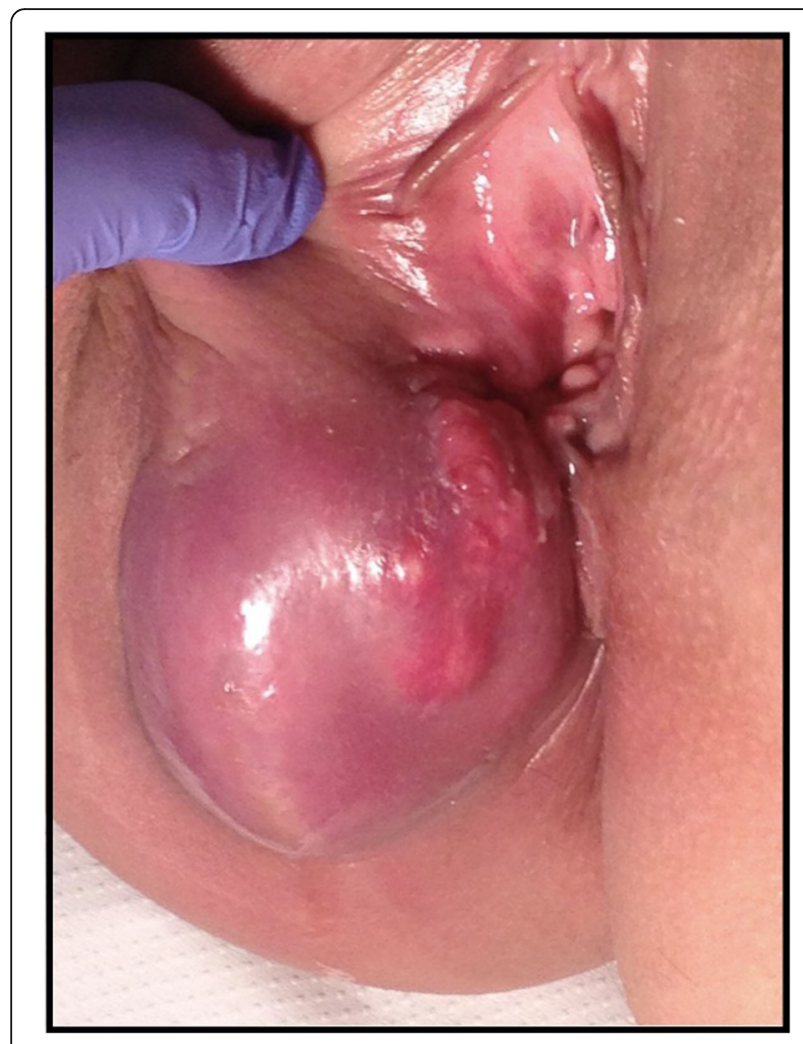

Fig. 1 Large metastasis located in the episiotomy scar site. The patient presented a chemoradioresistant cervical cancer diagnosed immediately after delivery. PE was performed with palliative aim

vulvar origin in 9 cases (39\%) and in the remaining 3 cases the tumour came from the endometrium (13\%).

In two cases, PE was performed with a palliative objective: in one case because of a rectal and vesical fistula to the vagina following chemo-radiotherapy for cervical cancer and in the other case due to a bulky metastasis of a cervical cancer located at the previous episiotomy scar site (Fig. 1).

\section{Surgical data}

In Table 2, surgical data are described. TPE was performed in 15 cases (65\%), followed by APE in 5 cases (22\%) and PPE in 3 cases (13\%). The operative time was $439 \pm 116$ min (range 210-690).

Colostomy was performed routinely in case of TPE and PPE (78\%). Up to $87 \%$ of the patients needed urinary diversion and Bricker was performed in all cases with one exception. This patient had only one kidney, and presented with renal failure due to severe ureteral obstruction after radiotherapy. The Bricker technique could not be accomplished and a nephrostomy was performed. In order to reach R0, pubic bone was removed in two cases due to tumour infiltration.

With regard to the perineal wound reconstruction, omentoplasty $(78 \%)$ was performed if feasible in combination with
Table 1 Baseline characteristics of the 23 study patients who underwent exenteration

\begin{tabular}{ll}
\hline Presentation of disease $[n(\%)]$ & \\
First diagnosis & $2(9)$ \\
Relapse & $19(82)$ \\
Persistence & $2(9)$ \\
Age $[$ Mean \pm SD (range)] & $57.8 \pm 12.4(29-84)$ \\
BMl [Mean \pm SD (range)] & $27.4 \pm 7.1(17-50)$ \\
Menopausal status $[n(\%)]$ & \\
Pre & $17(74)$ \\
Post & $6(26)$
\end{tabular}

ECOG performance status $[n(\%)]$

0

1

$2(9)$

2

1 (4)

Comorbidity $[n(\%)]$

None

Hypertension

Diabetes mellitus

Hypercholesterolemia

1 (4)

Monorenal

Primary tumour origin [n (\%)]

Vulvar cancer
Cervix cancer
Endometrial cancer

Previous treatment $[n(\%)]$

$\begin{array}{ll}\text { None } & 3(13) \\ \text { RT } & 4(17) \\ \text { ChT } & 0(0) \\ \text { RT + ChT } & 16(70)\end{array}$

Previous oncological surgery $[n(\%)]$

No

Yes

18 (78)

ASA score $[n(\%)]$

1

1 (4)

2

18 (78)

3

4 (17)

Operative intention $[n(\%)]$

Curative

Palliative

2 (9)

$B M I$ body mass index, ECOG Eastern Cooperative Oncology Group, $R T$ radiotherapy, ChT chemotherapy, ASA operative risk assessed according to the American Society of Anaesthesiology score

other techniques. Extended vertical rectus abdominis myocutaneous flap reconstruction (e-VRAM) was used in 30\% of the cases. In $57 \%$ of the cases (13 patients), primary closure was possible. A neo-vagina was created in four patients 
Table 2 Surgical data

\begin{tabular}{|c|c|}
\hline \multicolumn{2}{|l|}{ Type of exenteration $[n(\%)]$} \\
\hline Total & $15(65)$ \\
\hline Anterior & $5(22)$ \\
\hline Posterior & $3(13)$ \\
\hline \multicolumn{2}{|l|}{ Subtype of exenteration [n (\%)] } \\
\hline Type I (supralevator) & $5(22)$ \\
\hline Type II (infralevator) & $8(35)$ \\
\hline Type III (with vulvectomy) & $10(43)$ \\
\hline Operative time (min) [Mean \pm SD (range)] & $439 \pm 116(210-690)$ \\
\hline \multicolumn{2}{|l|}{ Subtype of reconstructive surgery [n (\%)] } \\
\hline Primary closure & $13(57)$ \\
\hline e-VRAM & $7(30)$ \\
\hline Neo-vagina & $4(17)$ \\
\hline Omentoplasty & $10(43)$ \\
\hline Gracilis flap & $1(4)$ \\
\hline V-Y flap & $2(9)$ \\
\hline \multicolumn{2}{|l|}{ Ostomy [n (\%)] } \\
\hline No & $5(22)$ \\
\hline Colostomy & $18(78)$ \\
\hline \multicolumn{2}{|l|}{ Urinary derivation $[n(\%)]$} \\
\hline No & $3(13)$ \\
\hline Bricker & $19(83)$ \\
\hline Nephrostomy & $1(4)$ \\
\hline \multicolumn{2}{|l|}{ Lymph node dissection $[n(\%)]$} \\
\hline No & $9(40)$ \\
\hline Inguinal & $4(17)$ \\
\hline Pelvic & $7(30)$ \\
\hline Paraortic & $6(26)$ \\
\hline \multicolumn{2}{|l|}{ Other procedures [n (\%)] } \\
\hline Pubic bone resection & $2(9)$ \\
\hline Mesh wall repair & $4(17)$ \\
\hline Intraoperative complications [n (\%)] & $1(4)$ \\
\hline Red blood units transfusion [Mean \pm SD (range)] & $0.96 \pm 1.15(0-5)$ \\
\hline Estimated blood loss (ml) [Mean \pm SD (range)] & $612 \pm 320(300-1300)$ \\
\hline \multicolumn{2}{|l|}{ Margin status $[n(\%)]$} \\
\hline RO & $21(91)$ \\
\hline$R<1$ & $2(9)$ \\
\hline
\end{tabular}

(17\%). Gracilis muscle flap was used in one case and $\mathrm{V}-\mathrm{Y}$ advancement flap in the other two patients. A free macroscopic intraoperative resection margin was obtained in all cases but tumor was found to affect the border in two cases (9\%). There was one major intraoperative complication (vascular injury) in which multiple transfusions were needed during repair.

\section{Complications}

Inpatient data and early complications are summarised in Table 3. The median hospital stay was 22.9 days (range 7-72) and the median stay in the intensive care unit (ICU) was 2.2 days (range 1-5). Four patients required some form of surgical re-operation. In two cases, a refresh of the abdominal wound edges and suturing was needed. One other patient presented a partial e-VRAM flap necrosis and reconstruction with a Gracilis flap was performed. In a fourth patient, a bilateral nephrostomy was needed due to renal failure and a non-functioning urinary diversion.

Early complications occurred in 17 patients (78\%). Grade II complication were common, affecting 15 patients (65\%), followed by 5 patients with grade III complications (22\%). Blood transfusion, total parenteral nutrition and wound or infectious complication constituted the majority of grade II events (Table 3). Two patients (9\%) presented grade IV complications, respiratory failure and sepsis, respectively, requiring admission to the ICU.

Data for medium-late complications are shown in Table 4 . Close to $2 / 3$ of the patients (15 cases, 65\%) needed readmission due to complications after discharge. Seven patients required some form of late re-surgery: in two cases, bilateral nephrostomy was needed due to renal failure and non-functioning urinary diversion. One patient presented with a small bowel leakage after Bricker and bowel resection was needed. One patient presented with a fistula of the uretero-ileal anastomosis (both bowel reservoir and ureters) so re-laparotomy was performed and a new Bricker was created. Another patient required a bowel resection due to an injury that occurred during the removal of a neo-vagina. This patient also presented with an acute limb ischaemia requiring multiple thrombectomies and endovascular stent emplacement. One patient presented with a prolapsed colostomy and a repair was performed and another patient presented with an abscess and surgical drainage was required.

Transfusion was needed in nine patients (39\%). Medium-late grade II complications occurred in 15 patients (65\%). Close to half of the sample (48\%) suffered grade III complications. The majority of the grade II and III complications were related to the urinary diversion procedure leading to pyelonephritis, sepsis, ureteral stricture or renal failure, requiring reallocation of ureteral catheters or nephrostomy. Four patients (18\%) presented severe complications (grade IV-V): 2 patients (9\%) were transferred to the ICU, due to coagulopathy and septic shock respectively, and the other 2 patients died as a consequence of these complications. The first patient presented with a pyelonephritis that developed into sepsis and multiple organ dysfunction syndrome before she died. The second patient presented with a bowel 
Table 3 Inpatient data and early complications

\begin{tabular}{|c|c|}
\hline Hospitalisation stay (days) [Mean \pm SD (range)] & $22.9 \pm 15.9(7-72)$ \\
\hline ICU stay $[n(\%)]$ & $2.2 \pm 1.5(1-5)$ \\
\hline Re- surgery $[n(\%)]$ & $4(17)$ \\
\hline $\operatorname{TPN}[n(\%)]$ & $10(43)$ \\
\hline Patients with transfusion [n (\%)] & $11(48)$ \\
\hline Transfusion (Units) [Mean \pm SD (range)] & $1.96 \pm 2.7(1-10)$ \\
\hline \multicolumn{2}{|l|}{ Circulatory event [n (\%)] } \\
\hline Thromboembolism & $0(0)$ \\
\hline Cardiovascular event & $0(0)$ \\
\hline \multicolumn{2}{|l|}{ Urinary $[n(\%)]$} \\
\hline No & $19(83)$ \\
\hline Ureter leakage & $2(9)$ \\
\hline Urostomy complication & $1(4)$ \\
\hline Renal failure & $3(13)$ \\
\hline \multicolumn{2}{|l|}{ Gastrointestinal $[n(\%)]$} \\
\hline Paralytic ileus & $6(26)$ \\
\hline Leakage & $0(0)$ \\
\hline \multicolumn{2}{|l|}{ Wound $[n(\%)]$} \\
\hline No & $15(65)$ \\
\hline Dehiscence & $7(30)$ \\
\hline Infection & $5(22)$ \\
\hline Haematoma & $1(4)$ \\
\hline Flap necrosis & $1(4)$ \\
\hline Lymphorrea & $1(4)$ \\
\hline \multicolumn{2}{|l|}{ Infectious $[n(\%)]$} \\
\hline No & $17(74)$ \\
\hline Abscess & $2(9)$ \\
\hline Fever and bacteremia & $3(13)$ \\
\hline Sepsis & $1(4)$ \\
\hline \multicolumn{2}{|l|}{ Respiratory [n (\%)] } \\
\hline Infectious & $0(0)$ \\
\hline Respiratory insufficiency & $1(4)$ \\
\hline \multicolumn{2}{|l|}{ Others $[n(\%)]$} \\
\hline Motor dysfunction & $1(4)$ \\
\hline \multicolumn{2}{|l|}{ Clavien Dindo [Mean \pm SD (range)] } \\
\hline Grade II & $1.8 \pm 1.6(0-5)$ \\
\hline Grade III & $0.3 \pm 0.5(0-2)$ \\
\hline Grade IV & $0.1 \pm 0.53(0-1)$ \\
\hline Grade V & $0 \pm(0-0)$ \\
\hline Patients with complication [n (\%)] & $5(22)$ \\
\hline Grade II & $15(65)$ \\
\hline Grade III & $5(22)$ \\
\hline Grade IV & $2(9)$ \\
\hline Grade V & $0(0)$ \\
\hline
\end{tabular}

ICU Intensive Care Unit, TPN total parenteral nutrition perforation requiring a large bowel resection. Later, the patient developed bowel and urinary fistulas from Bricker derivation and died as a consequence.

\section{Oncological outcome}

The OS and DFS results are shown in Fig. 2. The oncological outcomes are summarised in Table 5. Then, $<30$-day and $>$ 30-day mortality was $0 \%$ and $9 \%$ respectively: two patients died during follow-up due to medium-late complications related to the surgical procedure. The median follow-up was 18. 5 months (range 1-71). The OS after PE at 6 months, 1 year, 2 years and 4 years was $85 \%, 70.6 \%, 54.4 \%$ and $41.6 \%$ respectively. At the moment of writing, 14 patients $(61 \%)$ were alive with no evidence of disease, 8 patients (35\%) died of disease, 1 patient was alive with disease and no one died for other reasons. No lost in follow-up was recorded. The DFS at 6 months, 1 year, 2 years and 4 years after PE was $80 \%, 61.1 \%, 56.2 \%$ and $30.8 \%$ respectively.

\section{Discussion}

$\mathrm{PE}$ is the most radical procedure in cases of gynaecological malignancies. Its curative intention is based on locoregional control with complete excision of pelvic tumours and clear margins (R0). Five-year OS ranges between 30 and $60 \%[1,7,8]$. Our results were similar to the literature with an OS at 4 years of $41.6 \%$ and a mean follow-up period of 18.5 months.

In case of advanced primary tumours, the superiority of $\mathrm{PE}$ over radiotherapy or chemo-radiotherapy is not proven [9]. Exceptionally, in tumours such as sarcomas or melanomas which are known to be radiotherapy-resistant, PE can be a valid first option. In our study, two patients underwent PE as a primary treatment, one of them with a bulky episiotomy metastasis (Fig. 1) due to cervical neoplasia resistant to chemo-radiotherapy. The other one presented a vulvar cancer with anal sphincter involvement and refused chemo-radiotherapy.

Absolute contraindications to PE include fragile patients (poor performance status or medically unfit for surgery), bilateral sciatic nerve involvement and circumferential bone involvement [10]. Relative contraindications are extension of the tumour through the sciatic notch, encasement of external iliac vessels, irresectable distant metastases and predicted margin affected resection. In cases of encasement of external iliac vessels and pelvic wall tumour invasion, laterally extended endopelvic resection (LEER) and/or reconstruction of external iliac vessels can be performed to obtain free margins with good results [11].

Cervical cancer was the most common indication in the present study (48\%), followed by vulvar cancer (39\%) and endometrial cancer (13\%). In other studies, cervical cancer also represented the vast majority of cases $[8,12-15]$. Due to the tendency of ovarian cancer to 
Table 4 Medium-late complications

\begin{tabular}{|c|c|}
\hline Readmission [n (\%)] & $15(65)$ \\
\hline $\begin{array}{l}\text { Days after discharge [Mean } \pm \text { SD } \\
\text { (range)] }\end{array}$ & $102.4 \pm 136.2(10-515)$ \\
\hline Re- surgery $[n(\%)]$ & $7(30)$ \\
\hline Transfusion $[n(\%)]$ & $9(39)$ \\
\hline \multicolumn{2}{|l|}{ Circulatory event [n (\%)] } \\
\hline Thromboembolism & $1(4)$ \\
\hline Cardiovascular event & $0(0)$ \\
\hline \multicolumn{2}{|l|}{ Urinary $[n(\%)]$} \\
\hline No & $13(57)$ \\
\hline Pyelonephritis & $6(26)$ \\
\hline Fistula & $2(9)$ \\
\hline Ureteral stricture & $7(30)$ \\
\hline Bricker failure and reconstruction & $1(4)$ \\
\hline Renal failure & $4(17)$ \\
\hline \multicolumn{2}{|l|}{ Gastrointestinal $[n(\%)]$} \\
\hline No & $15(65)$ \\
\hline Paralytic ileus & $5(22)$ \\
\hline Small bowel fistula/perforation & $4(17)$ \\
\hline Eventration & $1(4)$ \\
\hline \multicolumn{2}{|l|}{ Infectious [n (\%)] } \\
\hline No & $13(57)$ \\
\hline Abscess & $4(17)$ \\
\hline Wound infection & $4(17)$ \\
\hline Sepsis & $6(26)$ \\
\hline \multicolumn{2}{|l|}{ Others $[n(\%)]$} \\
\hline Acute limb ischemia & $1(4)$ \\
\hline Coagulopathy & $1(4)$ \\
\hline Septic shock & $1(4)$ \\
\hline $\begin{array}{l}\text { Abdominal cloaca (Both ureter } \\
\text { and bowel fistula) }\end{array}$ & $1(4)$ \\
\hline \multicolumn{2}{|l|}{ Clavien Dindo [Mean \pm SD (range)] } \\
\hline Grade II & $1.4 \pm 1.4(0-4)$ \\
\hline Grade III & $1.1 \pm 1.5(0-6)$ \\
\hline Grade IV & $0.8 \pm 0.9(0-3)$ \\
\hline \multicolumn{2}{|l|}{ Patients with complications [n (\%)] } \\
\hline Grade II & $15(65)$ \\
\hline Grade III & $11(48)$ \\
\hline Grade IV & $3(13)$ \\
\hline Grade V & $2(9)$ \\
\hline
\end{tabular}

metastasise outside the pelvis, and also because of their sensitivity to adjuvant treatment, these patients are not usually good candidates for PE $[8,16]$.

The use of PE to control severe pelvic tumour symptoms in patients without a prospect of cure is controversial, even though it was described by Brunschwig as a purely palliative procedure [17]. PE was performed in two patients $(9 \%)$ with palliative indication in this study. Nevertheless, non-exenterative treatment such as chemotherapy, re-irradiation or analgesic infusion pumps should be offered as a first option if the objective is palliative care $[9,18,19]$.

Most of the complications after PE are related to the pre-irradiated tissue condition [15]. PE's postoperative mortality is described as less than $5 \%$, but with a high morbidity rate (above $50 \%$ ). In our patients, the observed mortality rate within 30 days was $0 \%$. A high morbidity rate is apparent not only in the early term (32\% of grade III-IV) but also in the medium to long term with a readmission rate of $65 \%$ frequently associated with complications related to the urinary tract.

To restore intestinal function, a permanent colostomy is usually performed. If anal sphincter preservation is achieved, a low colorectal/coloanal anastomosis to restore bowel continuity can be considered. It is associated with a high incidence of anastomotic leak (30-50\%) especially after previous full-dose pelvic RT. All cases of PPE or TPE in our study (78\%) received a permanent colostomy [20, 21].

To restore the urinary via, techniques can be classified into two groups: incontinent diversion (ileal conduits) or continent diversion (cutaneous reservoirs and orthotopic neobladder connected to urethra). Some authors concluded that there were no differences in postoperative complications between the two groups [7, 22]. Bricker was performed on almost $83 \%$ of our patients, producing a high rate of late complications including one patient requiring immediate postoperative reconstruction due to dehiscence. These results are similar to those in the literature where the overall complication rate of ileal conduits after pelvic radiation is described as being as high as 76\% [23].

The large perineal-pelvic floor defect created by $\mathrm{PE}$ usually needs to be filled in order to decrease postoperative complications and to achieve good functionality after surgery. Plastic reconstructive surgery by e-VRAM (30\%), Gracilis flap (4\%) and V-Y flap (9\%) achieved good results and restored the functionality of the pelvic floor. VRAM is the gold standard technique for reconstruction of large pelvic defects. However, we found that the e-VRAM provides skin coverage, reliability, good blood supply and tissue to fill the pelvic cavity after surgery [24, 25]. Despite the wide abdominal wall defect created and the presence of stomas, just one abdominal eventration was observed (colostomy site eventration) and by using this technique, we achieved a zero rate of pelvic eventration. The experience of the surgeon and the use of a mesh in all cases of e-VRAM probably contributed to this result. 


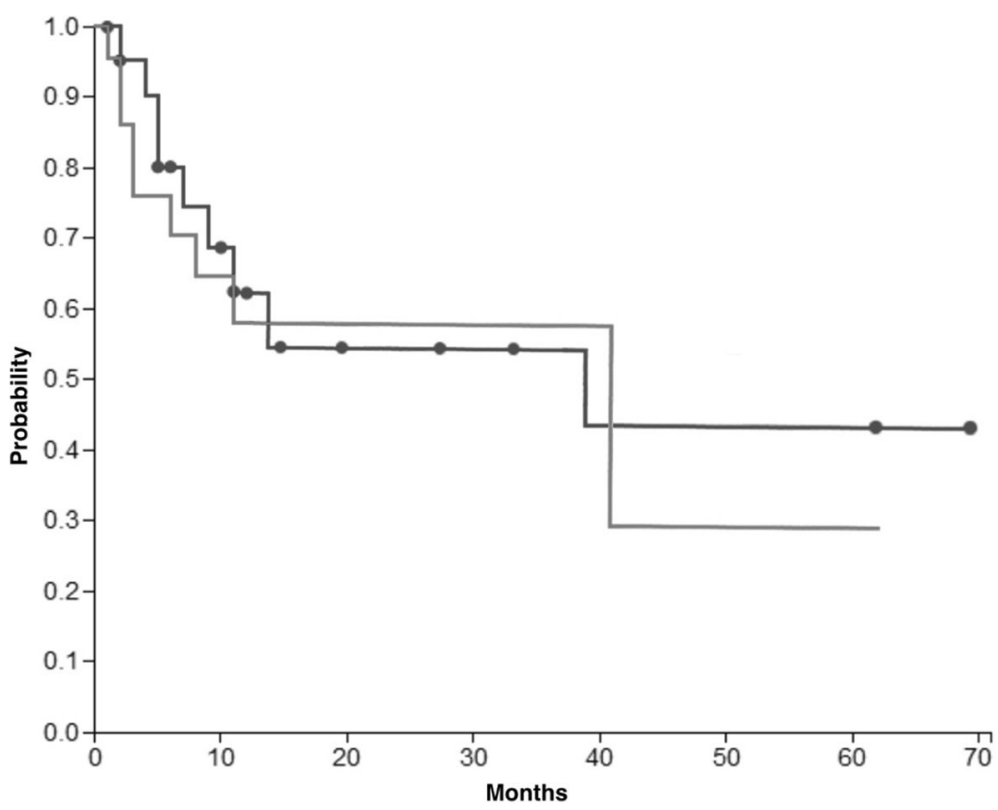

Fig. 2 Kaplan-Meier survival analysis curves. Overall survival (OS) in black and disease-free survival in grey (DFS)

Table 5 Oncological outcome

\begin{tabular}{ll}
\hline Mortality [n (\%)] & $0(0)$ \\
$<30$ days & $2(9)$ \\
$>30$ days & $18.5(1-71)$ \\
Follow-up [Mean (range)] & \\
OS (\%) & 85 \\
6 months & 70.6 \\
12 months & 54.4 \\
24 months & 41.6 \\
48 months & \\
DFS (\%) & 80 \\
6 months & 61.1 \\
12 months & 56.2 \\
24 months & 30.8 \\
48 months & \\
Disease status at last follow-up [n (\%)] & $14(61)$ \\
NED & $1(4)$ \\
AWD & $8(35)$ \\
DOD & $0(0)$ \\
DOC & $0(0)$ \\
\hline Lost in follow-up &
\end{tabular}

OS overall survival, DFS disease-free survival, NED no evidence of disease, AWD alive with disease, $D O D$ dead of disease: dead for other circumstances
Cases with involvement of the pelvic bone have classically been considered as inoperable, although Solomon et al. reported 37 cases in which it was required [26-29]. They achieved a $40 \%$ OS at 5 years compared with an OS of $4 \%$ for chemo-radiotherapy [26-29]. In our study, pubic bone resection was needed to achieve free margins in two cases, with no major complications associated with this technique [30].

Alterations in sexuality and changes in physical perception (deficits in self-image) are only a minor concern to patients before surgery, but they develop into the most important consequences of PE. Up to $17 \%$ of our patients had neo-vaginas constructed to support sexual function after surgery. Correct pre-surgery assessment, case evaluation by multidisciplinary boards and quality of life questionnaires should be mandatory in order to establish if patients are able to adapt to their new situation.

The limitations of this study are found in its retrospective nature, the small number of patients included, the limited follow-up, the lack of quality of life questionnaires and the heterogeneity of diagnosis for which PE was performed. These biases restricted extensive statistical analysis and results should be considered with caution. The majority of the studies reporting on PE in the literature are retrospective, so multi-centric prospective studies with increased numbers of patients should be performed.

\section{Conclusions}

In summary, pelvic exenteration provided approximately $40 \%$ chance of survival at 4 years in a selected 
group of patients with cancer of the low and middle female genital tract in which other therapy cannot be performed. PE can offer locoregional control in patients with no abdominal spread and where R0 resection is accomplished. It is important to make an exhaustive individual preoperative evaluation of the patient, highlighting the major consequences of this radical surgery.

\section{Acknowledgements}

The authors want to thank Ms. Sara García Álvarez for her helpful review and support in medical English editing during the writing of this article.

\section{Funding}

No funding.

\section{Availability of data and materials}

Data included in the study will be available at requirement

\section{Authors' contributions}

All authors contributed equally to the present manuscript. All authors read and approved the final manuscript.

\section{Authors' information}

Not applicable.

\section{Ethics approval and consent to participate}

The ethics committee approval was obtained before the performance of this study, and all patients consented to be included in or record for this purpose.

\section{Consent for publication}

They all consent for publication.

\section{Competing interests}

The authors declare that they have no competing interests.

\section{Publisher's Note}

Springer Nature remains neutral with regard to jurisdictional claims in published maps and institutional affiliations.

\section{Author details}

'Department of Gynecologic Oncology, University Hospital La Fe, Avinguda de Fernando Abril Martorell, 106, 46026 Valéncia. F Tower; 3rd Floor, Valencia, Spain. ${ }^{2}$ Department of Plastic Surgery, University Hospital La Fe, Valencia, Spain. ${ }^{3}$ Department of Surgery, University Hospital La Fe, Valencia, Spain. ${ }^{4}$ Department of Urology, University Hospital La Fe, Valencia, Spain.

Received: 26 June 2018 Accepted: 8 January 2019

Published online: 28 January 2019

\section{References}

1. Westin SN, Rallapali V, Fellman B, Urbauer DL, Pal N, Frumovitz MM et al (2014) Overall survival after pelvic exenteration for gynecologic malignancy. Gynecol Oncol 134(3):546-551

2. Fleisch MC, Pantke P, Beckmann MW, Schnuerch HG, Ackermann R, Grimm $\mathrm{MO}$ et al (2007) Predictors for long term survival after interdisciplinary surgery for advanced or recurrent gynecologic cancers. J Surg Oncol 95(6): 474-484

3. Devulapalli C, Jia Wei AT, DiBiagio J, Baez MI, Baltodano PA, Seal SM et al (2016) Primary versus flap closure of perineal defects following oncological resection. Plast Reconstr Surg 137:1602

4. Dessole M, Petrillo M, Lucidi A, Naldini A, Rossi M, De laco P et al (2018) Quality of life in women after pelvic exenteration for gynecological malignancies: a multicentric study. Int J Gynecol Cancer 28(2):267-273

5. Magrina JF (1990) Types of pelvic exenterations: a reappraisal. Gynecol Oncol 37(3):363-366
6. Dindo D, Demartines N, Clavien PA (2004) Classification of surgical complications: a new proposal with evaluation in a cohort of 6336 patients and results of a survey. Ann Surg 240:205-213

7. Moreno-Palacios E, Diestro MD, De Santiago J, Hernández A, Zapardiel I (2015) Pelvic exenteration in gynecologic cancer: La Paz University hospital experience. Int J Gynecol Cancer 25:1109-1114

8. Jäger L, Nilsson PJ, Rådestad AF (2013) Pelvic exenteration for recurrent gynecologic malignancy: a study of 28 consecutive patients at a single institution. Int J Gynecol Cancer 23:755-762

9. Höckel M, Dornhöfer N (2006) Pelvic exenteration for gynaecological tumours: achievements and unanswered questions. Lancet Oncol 7:837-847

10. Bhangu A, Beynon J, Brown G, Chang G, Das P, Desai A et al (2013) Consensus statement on the multidisciplinary management of patients with recurrent and primary rectal cancer beyond total mesorectal excision planes. Br J Surg 100(8):E1-33

11. Höckel M (2008) Laterally extended endopelvic resection (LEER)—principles and practice. Gynecol Oncol 111(2 Suppl):S13-S17

12. Petruzziello A, Kondo W, Hatschback SB, Guerreiro JA, Filho FP, Vendrame C et al (2014) Surgical results of pelvic exenteration in the treatment of gynecologic cancer. World J Surg Oncol 12:279

13. Maggioni A, Zanagnolo V, Peiretti M, Colombo N et al (2009) Pelvic exenteration: ten-year experience at the European Institute of Oncology in Milan. Gynecol Oncol 114(1):64-68

14. Kaur M, Joniau S, D'Hoore A, Van Calster B, Van Limbergen E, Leunen $\mathrm{K}$ et al (2012) Pelvic exenterations for gynecological malignancies: a study of 36 cases. Int J Gynecol Cancer 22:889-896

15. Chiantera V, Rossi M, De laco P, Koehler C, Marnitz S, Ferrandina G et al (2014) Survival after curative pelvic exenteration for primary or recurrent cervical cancer: a retrospective multicentric study of 167 patients. Int Gynecol Cancer 24(5):916-922

16. Van Wijk FH, van der Burg ME, Burger CW, Vergote I, van Doorn HC (2009) Management of recurrent endometrioid endometrial carcinoma: an overview. Int J Gynecol Cancer 19:314-320

17. Brunschwig A (1948|) Complete excision of pelvic viscera for advanced carcinoma; a one-stage abdominoperineal operation with end colostomy and bilateral ureteral implantation into the colon above the colostomy. Cancer 1:177-183

18. Brophy PF, Hoffman JP, Eisenberg BL (1994) The role of palliative pelvic exenteration. Am J Surg 167:386-390

19. Guimarães GC, Baiocchi G, Ferreira FO, Kumagai LY, Fallopa CC, Aguiar S et al (2011) Palliative pelvic exenteration for patients with gynecological malignancies. Arch Gynecol Obstet 283:1107-1112

20. Seagle BL, Dayno M, Strohl AE, Graves S, Nieves-Neira W, Shahabi S (2016) Survival after pelvic exenteration for uterine malignancy: a national cance data base study. Gynecol Oncol 143:472-478

21. Husain A, Curtin J, Brown C, Chi D, Hoskins W, Poynor E et al (2000) Continent urinary diversion and low-rectal anastomosis in patients undergoing exenterative procedures for recurrent gynecologic malignancies. Gynecol Oncol 78:208-211

22. Urh A, Soliman PT, Schmeler KM, Westin S, Frumovitz M, Nick AM et al (2013) Postoperative outcomes after continent versus incontinent urinary diversion at the time of pelvic exenteration for gynecologic malignancies. Gynecol Oncol 129:580-585

23. Hautmann RE, Hautmann SH, Hautmann O (2011) Complications associated with urinary diversion. Nat Rev Urol 8:667-677

24. Villa M, Saint-Cyr M, Wong C, Butler CE (2011 Jan) Extended vertical rectus abdominis myocutaneous flap for pelvic reconstruction: threedimensional and four-dimensional computed tomography angiographic perfusion study and clinical outcome analysis. Plast Reconstr Surg 127(1):200-209

25. Campbell CA, Butler CE (2011 Aug) Use of adjuvant techniques improves surgical outcomes of complex vertical rectus abdominis myocutaneous flap reconstructions of pelvic cancer defects. Plast Reconstr Surg 128(2):447-458

26. Milne t S mJ, lee P, Jm Y, Stalley P, Harrison JD (2013) Assessing the impact of a sacral resection on morbidity and survival after extended radical surgery for locally recurrent rectal cancer. Ann Surg 258:1007-1013

27. Dobrowsky W, Schmid AP (1985) Radiotherapy of presacral recurrence following radical surgery for rectal carcinoma. Dis Colon Rectum 28 917-919.1 
28. Milne t S mJ, Lee P, Young JM, Stalley P, Harrison JD et al (2014) Sacral resection with pelvic exenteration for advanced primary and recurrent pelvic cancer: a single-institution experience of 100 sacrectomies. Dis Colon Rectum 57:1153-1161

29. Solomon MJ, Tan KK, Bromilow RG, Al-mozany N, Lee PJ (2014) Sacrectomy via the abdominal approach during pelvic exenteration. Dis Colon Rectum 57:272-277

30. Solomon MJ, Austin KK, Masya L, Lee P (2015) Pubic bone excision and perineal urethrectomy for radical anterior compartment excision during pelvic exenteration. Dis Colon Rectum 58:1114-1119

Submit your manuscript to a SpringerOpen ${ }^{\circ}$ journal and benefit from:

- Convenient online submission

- Rigorous peer review

- Open access: articles freely available online

- High visibility within the field

- Retaining the copyright to your article

Submit your next manuscript at $\boldsymbol{\nabla}$ springeropen.com 